\title{
IONIZATION COOLING SCENARIO FOR A NEUTRINO FACTORY
}

\author{
R.C. Fernow, J.C. Gallardo*, R.B. Palmer, BNL, Upton, NY 11973, USA \\ P.L. Lebrun, FNAL, Batavia, IL 60510, USA
}

\begin{abstract}
The neutrino factory program $[1,2]$ aims to produce well-characterized neutrino fluxes, orders of magnitude larger than those available from conventional beams. An important feature of the machine design is a cooling section for reducing the muon transverse emittance to a level that can be accepted by the downstream accelerators and be contained in the storage ring. We describe simulations of a high-performance ionization cooling channel for the front end of a neutrino factory. The design considered here consists of a solenoidal lattice with alternating polarity and $2.75 \mathrm{~m}$ and $1.65 \mathrm{~m}$ cell lengths. Simulations show that the cooling increases the phase space density into the acceptance of the following linac by a factor of 3 .
\end{abstract}

\section{IONIZATION COOLING}

In ionization cooling, the beam loses both transverse and longitudinal momentum by ionization energy loss while passing through an absorber $[1,3]$. The longitudinal momentum is restored in rf accelerating cavities. This sequence, repeated many times, results in a reduction of the angular spread and thereby reduces the transverse emittance.

Ionization cooling is limited by multiple Coulomb scattering (MCS) in the absorbers. To minimize the MCS effect on cooling channel performance, we must have rather strong focusing at the absorber. Strong solenoidal fields are used for this purpose. Weak focusing leads to excessive emittance growth due to MCS. Very strong focusing is hard to achieve for a large aperture beam transport, and can also be detrimental to the $6 \mathrm{D}$ beam dynamics. As the beam divergence gets too large, the longitudinal velocity decreases too much, leading to a non-optimal longitudinal-transverse correlation factor and unacceptable growth of the longitudinal emittance. Choosing the right range of betatron function $\beta_{\perp, \min }$ with respect to the operating momentum is a key to a successful design $[1,4]$.

We have developed a number of tools for studying the ionization cooling process. Several tracking codes were written, or modified, to study the cooling process in detail. Two of these codes, ICOOL [5] and Geant4 [6], use Monte Carlo techniques to track particles through the cooling system. The codes include all relevant physical processes (e.g., energy loss, straggling, multiple scattering), and use electromagnetic fields that satisfy Maxwell's equations.

\footnotetext{
*gallardo@bnl.gov
}

\section{THE TAPERED SFOFO CHANNEL}

For optimal performance, the solenoidal field strength should be allowed to vary during the cooling process. To avoid the build up of canonical angular momentum, we flip the field while maintaining good focusing throughout the beam transport and low $\beta_{\perp}$ at the absorbers. One of the simplest solutions (the FOFO lattice [1, 3]) is to vary the field sinusoidally. The transverse motion in such lattices can be characterized by its betatron resonances, near which the motion is unstable. The stable operating region is between the low momentum $(2 \pi)$ and high momentum $(\pi)$ phase advance per half-period of the lattice. (Note that a half-period of the magnetic field lattice is one cell in our notation.)

The SFOFO lattice [2] is also based on the use of alternating solenoids, but adds a second harmonic to the simple sinusoidal field, producing an axial field similar to that shown in the lower part of Fig. 1. The axial field vanishes at the $\beta_{\perp, \min }$ position, located at the center of the absorber. This is accomplished by using two short focusing coils running in opposite polarity. However, unlike the FOFO case, the field decreases and flattens at $\beta_{\perp, \text { max }}$, due to a coupling coil located midway between the focusing coils, around the rf cavity. Figure 2 shows the design of a $2.75 \mathrm{~m}$ lattice cell. The transverse beam dynamics is strongly influenced by the solenoidal field profile on-axis and by the desired range of momentum acceptance.

This SFOFO lattice has several advantages over the FOFO. The focusing is approximately constant across the relevant momentum range, as we operate between the $2 \pi$ and $\pi$ resonances. Within this limited momentum range the transverse motion is stable. For a given $\beta_{\perp, \min }$, the SFOFO period is longer than the corresponding FOFO period, allowing longer absorbers per lattice cell and reducing the relative amount of multiple scattering in the absorber windows. The focusing coils can be located just around the absorbers, adjacent to the rf cavity. Since the absorber has a much smaller outer diameter than does the rf cavity, this arrangement allows the diameter of these high-field magnets to be reduced considerably.

For a given lattice period, one can adjust independently the location of the two betatron resonances, or, equivalently, the nominal operating momentum and the $\beta_{\perp, \text { min }}$ at that momentum. By adjusting these two parameters, we can keep the $\beta_{\perp}$ symmetric about the required nominal momentum, and independently reduce the central $\beta_{\perp}$ value. However, this is true over only a limited momentum range. As we decrease the coupling field and increase the focus- 

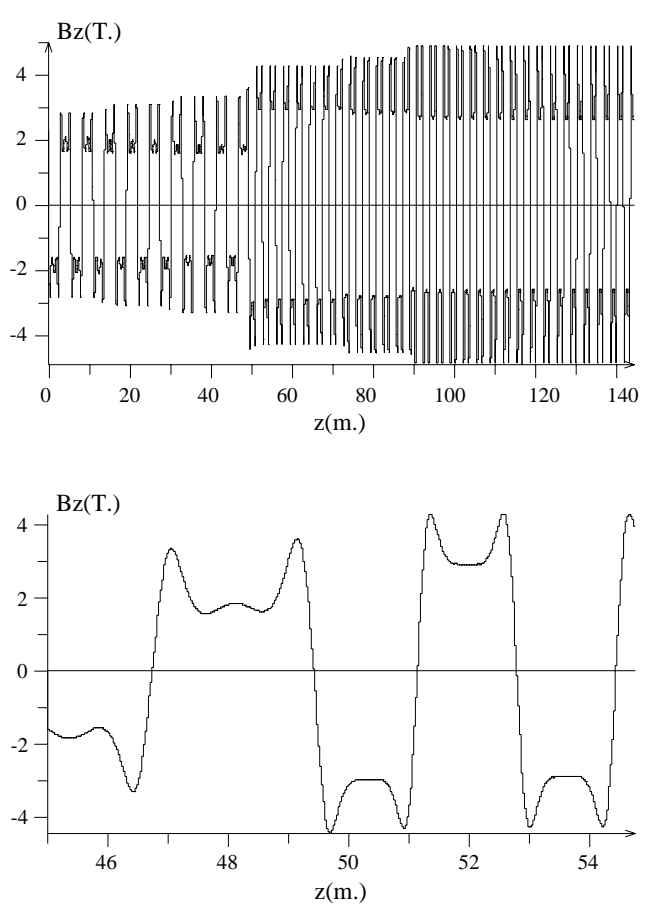

Figure 1: $B_{z}$ on axis for the entire SFOFO cooling channel (top); and for the matching section between $(1,3)$ and $(2,1)$ lattices (bottom).

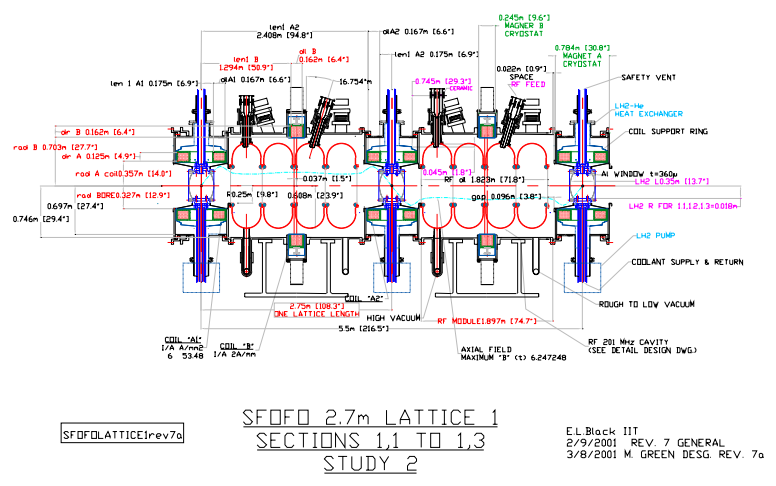

Figure 2: Engineering rendering of the $2.75 \mathrm{~m}$ cooling lattice cell.

ing field, the momentum acceptance will shrink as the $\pi$ and $2 \pi$ resonances move closer to the nominal momentum. At this point, we are forced to reduce the lattice period.

In addition the present design has $\beta_{\perp, \min }$ tapered along the cooling channel. One can slowly increase the focusing strength at a fixed operating momentum, while keeping a reasonable momentum acceptance. At a fixed $\beta_{\perp, \min }$, as the cooling progresses, the rms angle decreases. The cooling rate also decreases as the heating term due to multiple scattering becomes relatively significant. By slowly increasing the focusing strength (decreasing $\beta_{\perp, \text { min }}$ ), large rms angles $\left(\sigma_{x^{\prime}}=\sigma_{y^{\prime}} \approx 0.1 \mathrm{rad}\right)$ are maintained at the absorbers.

\section{DESCRIPTION OF THE CHANNEL}

The channel operates at a nominal momentum of 200 $\mathrm{MeV} / c$. There are six sections with decreasing $\beta_{\perp, \min }$. In the first three lattices, labeled $(1, \mathrm{i}), \mathrm{i}=1,3$, the lattice halfperiod (corresponding to one cell) is $2.75 \mathrm{~m}$, and in the other three lattices, $(2, \mathrm{i}), \mathrm{i}=1,3$, this half-period is $1.65 \mathrm{~m}$. A cell of the cooling lattice comprises one absorber, one linac and three coils. The matching sections between these sections also consist of cooling cells, which differ from the regular cooling cells only by the current circulating in the coils, with one exception: A different coil length must be used in the matching section between the $(1,3)$ and $(2,1)$ lattices. The total length of the cooling channel is $107.8 \mathrm{~m}$.

The lengths of the rf sections are constrained by the lattice design, as the focusing coils have a bore smaller than the rf cavities, and by the rf cell length, which must be optimized to give the high shunt impedance required to reach high gradient. In the simulations, cavities are always placed in the middle of the lattice cell. In order to improve the shunt impedance of the cavity, the iris of the cell is closed with a foil. Our baseline design calls for thin, prestressed beryllium windows with thicknesses that increase with radius. Closing the cavity iris with thin aluminum tubes arranged in a Cartesian grid has also been considered.

The absorber material is liquid hydrogen $\left(\mathrm{LH}_{2}\right)$. The absorber length is $35 \mathrm{~cm}$ for the (1,i), $\mathrm{i}=1,3$ lattices and 21 $\mathrm{cm}$ for the $(2, \mathrm{i})$ lattices, respectively. The muons therefore lose $\approx 12 \mathrm{MeV}$ per lattice cell for the $(1, \mathrm{i})$ lattices and $\approx 7 \mathrm{MeV}$ for the $(2, \mathrm{i})$ lattices, including the energy loss in the absorber windows. The $\mathrm{LH}_{2}$ vessels are equipped with thin aluminum windows. Window thicknesses are 360 (220) $\mu \mathrm{m}$, with radii of $18(11) \mathrm{cm}$, for the $(1, \mathrm{i})$ and $(2, \mathrm{i})$ lattices, respectively.

\section{PERFORMANCE}

The beta function and beam radius step down with each new section of the cooling lattice. The beam divergence at the absorbers is kept essentially constant in order to minimize the effects of multiple scattering. The $\beta_{\perp, \text { min }}$ function, derived from the beam second-order moments at the absorber centers, varies from $47 \mathrm{~cm}$ to $18 \mathrm{~cm}$ over the length of the channel.

The transverse emittance cools from 12 to $\approx 2 \mathrm{~mm}$-rad. The longitudinal emittance remains more or less stable, at around $30 \mathrm{~mm}$. This is somewhat deceptive. The antidamping slope of the energy loss curve, straggling, and imperfections in the longitudinal-to-transverse correlation cause particles to fall out of the rf bucket and to be scraped away due to the strong betatron resonances, as seen in the top curve of Fig. 3. In fact, the buncher delivers a full rf bucket to the cooling section and the longitudinal emittance cannot grow any larger. This scraping occurs on the com- 


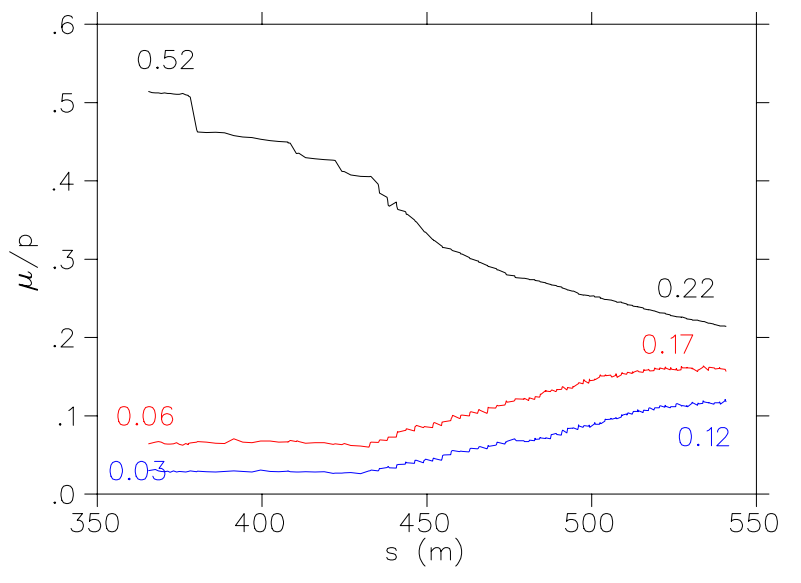

Figure 3: Particle transmission in the buncher and cooling sections. Top curve is overall muons per incident proton; middle curve is yield into Feasibility Study-II acceptance; lower curve is yield into Feasibility Study-I acceptance.

bined time scales of the synchrotron period, about $20 \mathrm{~m}$, and the growth time of the betatron resonance instabilities.

Despite this overall loss, the number of particles within the accelerator acceptance increases. The lower two curves in Fig. 3 give the number of particles within the baseline longitudinal and transverse acceptance cuts. The middle curve represents the values for the accelerator parameters assumed in this study, Feasibility Study-II [2] (15 mm-rad transverse acceptance). The lower curve, shown for comparison, gives the values for the acceptances used in Feasibility Study-I [1] (9.35 mm-rad transverse acceptance). Both studies used $150 \mathrm{~mm}$ longitudinal acceptance. It is seen that the gain in muons due to cooling within the accelerator acceptance is a factor of $\approx 3$ (or $\approx 4$ if the Study-I acceptances were used). If the particle loss from longitudinal emittance growth could be eliminated, as might be the case if emittance exchange were used [3], then these gains could double.

The performance of the cooling channel is limited by both multiple scattering and the momentum acceptance. While the latter is difficult to estimate based on computer simulations, it is straightforward to estimate the multiple scattering by simply turning the effect off in the codes. Without multiple scattering, the $\mu / P_{15}$ and $\mu / P_{9.35}$ yields would increase by relative amounts of $20 \%$ and $40 \%$, respectively.

\section{TOLERANCES \& SYSTEMATICS}

Since an ionization cooling channel has never been built, the issue of how sensitive the performance values are to small changes in the model assumptions is an important question. Among the issues that we have considered [2] are sensitivity to the multiple scattering model, control of the energy loss in $\mathrm{LH}_{2}$, control of the energy gain in the linac, magnet alignments, and space charge. In addition, detailed comparisons of the simulation model were made with the engineering model, which takes into account constraints from the solenoid magnet subsystem, rf cavities, absorbers and diagnostics. The basic dimensions of the cooling cells were taken from the conceptual design. However, the exact placement of various components departed slightly from the layout used in the simulations. Items that have been examined [2] are coil positions, sizes and current densities, the effect of split coil blocks, absorber window shape, and tapered thickness rf-window designs. None of these modifications had a significant effect on the expected channel performance.

\section{ACKNOWLEDGEMENTS}

We would like to thank D. Kaplan, E. Black, Y. Torun and G. Penn for contributions to these studies. This research was supported by the U.S. Department of Energy under contracts DE-AC02-98CH10886, DE-AC0276CH03000 and DE-AC03-76SF00098.

\section{REFERENCES}

[1] Feasibility Study-I; A Neutrino Source Based on an Intense Muon Storage Ring, eds. N. Holtkamp and D. Finley, Fermilab-Pub-00/108-E (2000); available at http://www.fnal.gov/projects/muon_collider/nu-factory/nufactory.html

[2] Feasibility Study-II of a Muon-Based Neutrino Source, eds. S. Ozaki, R. Palmer, M. Zisman and J. Gallardo, BNL-52623 (2001); available at http://www.cap.bnl.gov/mumu/studyii/final_draft/

[3] C.M. Ankenbrandt et al., Phys. Rev. ST Accel. Beams 2, 081001 (1999).

[4] J. Monroe et al. Design and Simulation of Muon Ionization Cooling Channels for the Fermilab Neutrino Factory Feasibility Study, Phys. Rev. ST Accel. Beams 4, 041301 (2001).

[5] R. Fernow, ICOOL, A Simulation Code for Ionization Cooling of Muons Beams, Proceedings of the 1999 Particle Accelerator Conference, A. Lucio and W. MacKay, eds. (IEEE, Piscataway, NJ, 1999) p. 3020.

[6] The Geant4 Tool Kit is available at http://wwwinfo.cern.ch/asd/geant4/geant4.html. 